\title{
Mechanism of Long Noncoding RNA HOTAIR in Nucleus Pulposus Cell Autophagy and Apoptosis in Intervertebral Disc Degeneration
}

\author{
Shujun Zhang $\mathbb{D}^{1,2}$ Sheng Song, ${ }^{1}$ Wei Cui, ${ }^{2}$ Xueguang Liu, ${ }^{1}$ and Zhenzhong Sun ${ }^{1}{ }^{1}$ \\ ${ }^{1}$ Department of Spine Surgery, Wuxi 9th Affiliated Hospital of Soochow University, Suzhou, China \\ ${ }^{2}$ Department of Minimally Invasive Spine Surgery, Wuhan Pu'ai Hospital, Wuhan, China \\ Correspondence should be addressed to Shujun Zhang; zshujun0415@163.com and Zhenzhong Sun; jinping924965@163.com
}

Received 5 August 2021; Revised 3 November 2021; Accepted 22 November 2021; Published 3 January 2022

Academic Editor: Sakineh Kazemi Noureini

Copyright (C) 2022 Shujun Zhang et al. This is an open access article distributed under the Creative Commons Attribution License, which permits unrestricted use, distribution, and reproduction in any medium, provided the original work is properly cited.

Objective. Intervertebral disc degeneration (IDD) contributes to cervical and lumbar diseases. Long noncoding RNAs (lncRNAs) are implicated in IDD. This study explored the mechanism of lncRNA HOTAIR in IDD. Methods. Normal and degenerative nucleus pulposus (NP) cells were isolated from NP tissues obtained in intervertebral disc surgery. Cell morphology was observed by immunocytochemistry staining and toluidine blue staining. NP cell markers were detected by RT-qPCR. Proliferation was detected by MTT assay. Autophagy-related proteins were detected by Western blot. Autophagosome was observed by monodansylcadaverine fluorescence staining. Apoptosis was detected by TUNEL staining and flow cytometry. si-HOTAIR and/or miR148a inhibitor was introduced into degenerative NP cells. Binding relationships among HOTAIR, miR-148a, and PTEN were predicted and verified by dual-luciferase reporter assay and RNA pull-down. Finally, IDD rat models were established. Rat caudal intervertebral discs were assessed by HE staining. Expressions of HOTAIR, miR-148a, and PTEN were determined by RT-qPCR. Results. HOTAIR was highly expressed in degenerative NP cells $(p<0.05)$. si-HOTAIR inhibited degenerative NP cell apoptosis and autophagy $(p<0.05)$. HOTAIR upregulated PTEN as a sponge of miR-148a. miR-148a was poorly expressed in degenerative NP cells. miR-148a deficiency partially reversed the inhibition of si-HOTAIR on degenerative NP cell autophagy and apoptosis (all $p<0.05)$. In vivo assay confirmed that si-HOTAIR impeded autophagy and apoptosis in intervertebral disc tissues, thus improving pathological injury in IDD rats (all $p<0.05$ ). Conclusion. LncRNA HOTAIR promoted NP cell autophagy and apoptosis via promoting PTEN expression as a ceRNA of miR-148a in IDD.

\section{Introduction}

Intervertebral discs (IVDs) are composed of nucleus pulposus (NP), annulus fibrosus (AF), and cartilage endplates, which experience a gradual degeneration under the influence of a variety of factors, such as aging and tissue damage caused by mechanical stress [1, 2]. IVD degeneration (IDD), considered a prevailing public health problem, severely affects patients' quality of life and poses huge economic burdens to families and society $[3,4]$. As a highly prevalent musculoskeletal disease, IDD is associated with back and neck pain and nerve root-related pain, featured by progressive degenerative injury of intervertebral tissues and metabolic changes in other vertebral tissues [5]. The reduction of NP cell numbers, for example, in the form of apoptosis and autophagy, is tightly implicated in IDD pathogenesis [4]. Currently, the management options for IDD are primarily aimed at relieving symptoms, and therapeutic interventions involving the underlying pathology of IDD are not available [6]. Therefore, this paper sought to find novel and effective treatment approaches to improve IDD therapeutic effects.

Long noncoding RNAs (lncRNAs) are characterized by limited protein-coding ability and are involved in multiple biological processes, such as transcription, protein activity, and aging-related degenerative musculoskeletal diseases, including IDD [7]. Deregulated $\ln c$ RNAs play a principal part in modulating NP cell behaviors in IDD [8]. HOX transcript antisense intergenic RNA (HOTAIR) is a kind of lncRNA associated with autophagy [9]. Meanwhile, 
autophagy is validated to promote IDD [10]. HOTAIR has been repeatedly proven to facilitate NP cell apoptosis and senescence by stimulating autophagy $[10,11]$. Mechanically, growing studies have proposed that $\ln \mathrm{R}$ NAs interact with microRNAs (miRNAs), thereby exerting effects on cell autophagy and apoptosis via a lncRNA-miRNA-mRNA competing endogenous RNA (ceRNA) network [12, 13]. For example, IncRNA FAM83H-AS1 can promote the growth of NP cells and maintain the tissue homeostasis of IVDs by inhibiting miR-22-3p [14]. LncRNA H19 can promote autophagy and apoptosis of NP cells, thus aggravating IDD via the miR-139/CXCR4/NF- $\kappa$ B axis [15]. LncRNA CDKN2B-AS1 can inhibit ox-LDL-induced proliferation of vascular smooth muscle cells and promote apoptosis through the ceRNA network of CDKN2B-AS1/miR-126-5p/ PTPN7 [16].

miRNAs, defined as highly conserved molecules, show deregulated expression in diverse musculoskeletal diseases, including IDD, and hold the promise as reliable biomarkers for IDD [17]. Several miRNAs have been demonstrated to inhibit NP cell autophagy and apoptosis in IDD, which can be mediated by lncRNAs $[18,19]$. For instance, miR-202-5p can affect the autophagy and apoptosis of degenerative NP cells by targeting ATG7 [18]. LncRNA H19 promotes NP cell autophagy and apoptosis and aggravates IDD through the miR-139/CXCR4/NF- $\kappa$ B axis [15]. miR-32-5p mimic contributes to NC cell proliferation and decreases apoptosis by upregulating the PI3K/AKT/mTOR pathway [20]. Additionally, a previous study has proposed that miR-148a shows intimate associations with IDD pathogenesis and close interactions with NP cells [21]. Nevertheless, little is known about the effect of miR-148a on NP cell autophagy and apoptosis in IDD.

Therefore, it is reasonable to speculate that lncRNA HOTAIR may play a role in NP cell autophagy and apoptosis with the involvement of miR-148a in IDD. Consequently, we performed a series of histological and molecular experiments to identify the underlying ceRNA network of IncRNA HOTAIR-miR-148a-mRNA in IDD, with the purpose of providing some novel therapies against $\mathrm{AD}$.

\section{Materials and Methods}

2.1. Ethics Statement. All experimental protocols were recommended and got approval from the Ethics Committee of Wuxi 9th Affiliated Hospital of Soochow University (approval number: KT2019016). The experimental process strictly followed the approved protocol. Significant efforts were made to minimize animal numbers and suffering. Consent from patients and the Ethics Committee of our hospital was obtained prior to sample collection.

2.2. Isolation and Culture of NP Cells. IVDs used in this study were collected from the clinical operations in the Orthopedics Department of our hospital. IDD was assessed using T2-weighted sagittal magnetic resonance imaging (MRI) according to the modified Pfirrmann grading system before operation [22]. Based on MRI results, nondegenerative NP tissues were collected from 5 patients ( 3 males and 2 females; aged 12-20 years; Pfirrmann grade I) undergoing fusion surgery for adolescent idiopathic scoliosis or lumbar vertebral burst fractures, whereas severely degenerative NP tissues were collected from 5 lumbar disc herniation patients (3 males and 2 females; aged 45-58 years; Pfirrmann grades IVV) undergoing discectomy surgery. The collected NP tissues were cut into small pieces using aseptic ophthalmic scissors and detached using phosphate-buffered saline (PBS) containing $0.025 \%$ type I collagenase (Invitrogen Inc., Carlsbad, CA, USA) for $4 \mathrm{~h}$, followed by $10 \mathrm{~min}$ centrifugation. Next, the cell precipitates at the bottom of the centrifuge tube were dispersed and mixed. The obtained cell suspension was transferred into the culture bottle for culture. The primary NP cells were cultured in Dulbecco's modified Eagle's medium/ F12 (1:1) medium (Gibco Company, Grand Island, NY, USA) with $10 \%$ fetal bovine serum (Gibco) in an incubator $\left(37^{\circ} \mathrm{C}, 5 \% \mathrm{CO}_{2}\right)$. For about 7 days, the medium was refreshed for the first time upon complete cell adherence to the wall. When the primary cultured cells covered the monolayer (over 95\% confluence), they were subcultured for phenotypic identification. An inverted phase-contrast microscope (Olympus Optical Co., Ltd., Tokyo, Japan) was used to observe cell morphology after $48 \mathrm{~h}$, and NP cells of P2 generation were selected for subsequent experiments.

2.3. Cell Transfection. Small-interfering (si)-HOTAIR, sinegative control (NC), mimic NC, miR-148a mimic, inhibitor NC, and miR-148a inhibitor were supplied by Sangon Biotech Co., Ltd. (Shanghai, China). All transfections were conducted following Lipofectamine 2000 (Invitrogen) instructions. Cells were cultured in an incubator for subsequent experiments.

2.4. Immunocytochemistry (ICC) Staining. After cleaning, acid soaking, and sterilizing, the cover slides were placed in the cell culture dish and added with cell suspension for 24-48 h conventional culture. After the cover slides were covered by cells, the culture medium was removed. Then cells were rinsed with PBS, fixed in $4 \%$ formaldehyde for $15 \mathrm{~min}$, and incubated with $0.3 \%$ Triton- 100 for $20 \mathrm{~min}$. Following PBS washing, the cells underwent $30 \mathrm{~min}$ incubation with $1 \%$ bovine serum albumin (BSA) and then $1 \mathrm{~h}$ incubation with primary antibody Collagen II $(1: 500$, ab34712, Abcam Inc., Cambridge, MA, USA) at $37^{\circ} \mathrm{C}$. Following PBS washing, the cells were reacted with secondary antibody immunoglobulin G (IgG) $(1: 2000$, ab205718, Abcam) for $30 \mathrm{~min}$ in a wet box, followed by PBS washing. Next, the cells were conventionally stained with 2,4-diaminobutyric acid and counterstained with hematoxylin. Following dehydration and clearance, the cells were sealed and observed under a microscope (TS100, Nikon Instruments, Chiyoda-ku, Tokyo, Japan).

2.5. Toluidine Blue Staining. NP cells underwent 2 min PBS washing and $30 \mathrm{~min}$ fixing with $4 \%$ paraformaldehyde room temperature, followed by PBS washing. Then the cells were 
immersed and stained with $1 \%$ toluidine blue (SigmaAldrich, Merck KGaA, Darmstadt, Germany) for $2 \mathrm{~h}$ and counterstained with hematoxylin. Next, the excess staining solution was removed using 95\% ethanol. Finally, the cells were cleared in xylene, sealed using neutral gum, and observed and photographed under a microscope.

2.6. 3-(4,5-Dimethylthiazol-2-yl)-2,5-diphenyltetrazolium Bromide (MTT) Assay. Cells in the exponential phase of growth were harvested and added with $5 \mathrm{~g} / \mathrm{L}$ MTT solution (20 $\mu \mathrm{L}, \mathrm{Gibco})$ at $24 / 48 / 72 / 96 \mathrm{~h}$ of culture, respectively. After $4 \mathrm{~h}$ further culture and the removal of the supernatant, each well was added with $150 \mu \mathrm{L}$ dimethyl sulfoxide, followed by shaking for full dissolution of crystals. Next, the optical density (OD) value at $490 \mathrm{~nm}$ of each well was determined using a microplate reader (Rayto Life Science Co., Ltd., Shenzhen, Guangdong, China), with 5 duplicated wells for each group. The experiment was repeatedly performed three times.

\subsection{Reverse Transcription Quantitative Polymerase Chain} Reaction (RT-qPCR). Total RNA was extracted using TRIzol (Invitrogen) one-step method, and high-quality RNA was verified using UV spectrophotometer and formaldehyde denaturation electrophoresis. The cDNA was synthesized through reverse transcription from $1 \mu \mathrm{g}$ RNA using AMV reverse transcriptase. SYBR Green Mix (Thermo Fisher Scientific Inc., Waltham, MA, USA) was used for qPCR. PCR primer design and synthesis were accomplished by Sangon (Table 1). The reaction system included cDNA $(1.0 \mu \mathrm{L})$, $2 \times$ SYBR Green Mix $(10 \mu \mathrm{L})$, forward primer $(10 \mu \mathrm{M}$; $0.5 \mu \mathrm{L})$, and reverse primer $(10 \mu \mathrm{M} ; 0.5 \mu \mathrm{L})$, and RNase free water was used to supplement to $20 \mu \mathrm{L}$. Reaction conditions were $94^{\circ} \mathrm{C}$ for $5 \mathrm{~min}$ (predenaturation) and then 40 cycles of $94^{\circ} \mathrm{C}$ for $40 \mathrm{~s}$ (denaturation), $60^{\circ} \mathrm{C}$ for $40 \mathrm{~s}$ (annealing), and $72^{\circ} \mathrm{C}$ for $1 \mathrm{~min}$ (extending), followed by $10 \mathrm{~min}$ extending at $72^{\circ} \mathrm{C}$. U6 served as an internal parameter for miR-148a, and glyceraldehyde-3-phosphate dehydrogenase (GAPDH) served as an internal parameter for HOTAIR, aggrecan, Collagen II, and PTEN. The $2^{-\Delta \Delta \mathrm{Ct}}$ method was used for data analysis.

2.8. Western Blot (WB) Analysis. Cell protein was extracted, and protein concentration was determined as per the instructions of the bicinchoninic acid kit (Boster Biological Technology Co., Ltd., Wuhan, Hubei, China). Following the addition of loading buffer $(30 \mu \mathrm{g} / \mathrm{well})$, the extracted protein underwent $10 \mathrm{~min}$ boiling at $95^{\circ} \mathrm{C}$. The protein was then subjected to $10 \%$ polyacrylamide gel (Boster) electrophoresis (voltage changed from $80 \mathrm{~V}$ to $120 \mathrm{~V}$ ) for separation and then loaded onto polyvinylidene fluoride membranes via wet transfer at a voltage of $100 \mathrm{mV}$ for $45-70 \mathrm{~min}$. Following $1 \mathrm{~h}$ blocking with $5 \%$ BSA at room temperature, the membranes underwent overnight incubation at $4^{\circ} \mathrm{C}$ with primary antibodies phosphatase and tensin homolog (PTEN) $(1: 1000$, ab267787, Abcam), light chain 3 (LC3)I, LC3II (ZSGB-Bio Co., Ltd, Beijing, China), p62 (2 $\mu \mathrm{g} / \mathrm{mL}$, ab56416, Abcam),
Table 1: Primer sequences for RT-qPCR.

\begin{tabular}{lc}
\hline Gene & Primer \\
\hline \multirow{2}{*}{ HOTAIR } & F: $5^{\prime}$-CATTCTGCCCTGATTTCCG-3' \\
& R: $5^{\prime}$-ATCCGTTCCATTCCACTGCG-3' \\
\hline \multirow{2}{*}{ miR-148a } & F: $5^{\prime}$-ATGCTCAGTGCACTACAGAA-3' \\
& R: $5^{\prime}$-GTGCAGGGTCCGAGGT-3' \\
\hline \multirow{2}{*}{ Aggrecan } & F: $5^{\prime}$-TGAGCGGCAGCACTTTGAC-3' \\
& R: $5^{\prime}$-TGAGTACAGGAGGCTTGAGG-3' \\
\hline \multirow{2}{*}{ Collagen II } & F: $5^{\prime}$-TCCAGATGACCTTCCTACGC-3' \\
& R: $5^{\prime}$-TCCAGATGACCTTCCTACGC-3' \\
\hline \multirow{2}{*}{ PTEN } & F: $5^{\prime}$-CCATAACCCACCACAG-3' \\
& R: $5^{\prime}$-CAGTCCGTCCTTTC-3' \\
\hline \multirow{2}{*}{ GAPDH } & F: $5^{\prime}$-GGGAGCCAAAAGGGTCAT-3' \\
\hline \multirow{2}{*}{ U6 } & R: $5^{\prime}$-GAGTCCTTCCACGATACCAA-3' \\
\hline
\end{tabular}

Beclin-1 (1 :2000, ab207612, Abcam), and GADPH (1:2500, ab9485, Abcam). Following 3 washes with Tris-buffered saline-Tween 20 ( $5 \mathrm{~min}$ per wash), the membranes underwent $1 \mathrm{~h}$ incubation with a secondary antibody $\operatorname{IgG}(1: 2000$, ab205718, Abcam) at room temperature. The membranes were washed ( 3 times, $5 \mathrm{~min}$ each) and developed using chemiluminescence reagent and GelDOC EZ Imager (BioRad Inc., Hercules, CA, USA). ImageJ software (National Institutes of Health, Bethesda, Maryland, USA) was utilized to analyze the gray value of the target bands.

2.9. Monodansylcadaverine (MDC) Fluorescence Staining. NP cells in each group underwent $4 \mathrm{~h}$ culture in an incubator $\left(37^{\circ} \mathrm{C}, 5 \% \mathrm{CO}_{2}\right)$. After medium removal, the cells were rinsed twice with $\mathrm{PBS}$ and added with MDC staining solution (0.1 mM, Sigma-Aldrich) for $45-60 \mathrm{~min}$ incubation at $37^{\circ} \mathrm{C}$. After the removal of the MDC staining solution, the cells were subjected to 15 min fixing at room temperature in $4 \%$ paraformaldehyde and 2 washes with PBS. Next, cells were observed and photographed using a fluorescence microscope (excitation wavelength $=425 \mathrm{~nm}$; emission wavelength $=525 \mathrm{~nm}$ ) after drying. A total of 100 cells were randomly selected in the field $(200 \times)$, and the number of cells containing punctate fluorescent autophagic vesicles was counted (the NP cells with 5 or more punctate fluorescent autophagic vesicles were counted once). The autophagy percentage $=$ cells containing autophagic vesicles $/ 100$ cells $\times 100 \%$. The final value was the average of three repeated tests.

2.10. Terminal Deoxynucleotidyl Transferase- (TdT-) Mediated dUTP Nick End Labeling (TUNEL) Staining. The cells $\left(1 \times 10^{5} /\right.$ well $)$ were seeded into 6 -well plates and cultured for $24 \mathrm{~h}$. After stable attachment to the slide, the cells underwent PBS washing and 30 min fixing with $4 \%$ paraformaldehyde, followed by $30 \mathrm{~min}$ incubation with $0.1 \%$ Triton X-100 solution. Based on the instructions of a TUNEL kit, the enzyme reaction solution (tube 1) and the labeling solution (tube 2) $(1: 9)$ were added to the cover slides, with the NC group only 
added with the labeling solution (tube 2) as control. Next, the cells underwent 60 min incubation away from light at $37^{\circ} \mathrm{C}$ under saturated humidity. Hoechst working solution was added to stain the nucleus for $20 \mathrm{~min}$ incubation at room temperature. Finally, cover slides were sealed using an antifade mounting medium, and a fluorescence microscope (Olympus) was utilized for cell apoptosis observation.

2.11. Flow Cytometry. The cells were detached using trypsin and made into a single-cell suspension. After centrifugation $\left(4^{\circ} \mathrm{C}, 100 \mathrm{~g}, 5 \mathrm{~min}\right)$ and the supernatant removal, the cells were washed twice with precooled PBS, suspended in the binding buffer, and added with $5 \mu \mathrm{L}$ Annexin $\mathrm{V}$ and $1 \mu \mathrm{L}$ propidium iodide working solution for $15 \mathrm{~min}$ reaction at room temperature avoiding light. Next, $300 \mu \mathrm{L}$ binding buffer was added and gently mixed. Approximately 10000 cells were determined utilizing a flow cytometer, and then the percentage of apoptotic cells was calculated.

2.12. RNA-Fluorescence In Situ Hybridization (FISH). The subcellular localization of lncRNA HOTAIR in NP cells was determined with firm compliance to the instructions of the RNAscope Multiplex Assay kit (Thermo Fisher). The HOTAIR fluorescent probe was designed and provided by the kit's manufacturer [23].

2.13. Nuclear and Cytoplasmic Fractionation Assay. The nuclear extract was prepared based on the manufacturer's instructions using the NE-PER nuclear and cytoplasmic extraction kit (Thermo Fisher). Cell precipitates were subjected to vortex oscillation for $15 \mathrm{~s}$ and suspended in $1 \mathrm{~mL}$ cytosol extraction reagent (CER)I (10 times the volume of cell precipitation) supplemented with phenylmethylsulfonyl fluoride (PMSF). Next, the suspension was incubated on ice for $10 \mathrm{~min}$ and then added with CERII (CERI: CERII: nuclear extraction reagent $($ NER $)=200: 11: 100)$ for $5 \mathrm{~s}$ vortex oscillation, followed by $1 \mathrm{~min}$ incubation on ice and centrifugation $(16000 \mathrm{~g}, 5 \mathrm{~min})$. The supernatant (cytoplasmic extract) was transferred to the precooled tube. The insoluble precipitates containing coarse nuclei underwent $15 \mathrm{~s}$ vortex oscillation and resuspension in $1 \mathrm{~mL}$ NER added with PMSF (PMSF: $\mathrm{NER}=1: 100$ ), followed by $40 \mathrm{~min}$ incubation on ice. Vortex was carried out for $15 \mathrm{~s}$ every $10 \mathrm{~min}$. After centrifugation $(16000 \mathrm{~g}, 10 \mathrm{~min})$, the obtained supernatant was nuclear extract. HOTAIR expression in nuclear and cytoplasmic extracts was detected using RT-qPCR.

2.14. Dual-Luciferase Reporter Gene Assay. Bioinformatics software and websites were utilized to predict the binding sites of HOTAIR with miR-148a and miR-148a with PTEN. HOTAIR $3^{\prime}$ UTR and PTEN $3^{\prime}$ UTR sequences containing miR-148a binding site were synthesized, and HOTAIR-wild type (WT) and PTEN-WT plasmids were constructed. Based on these plasmids, the binding sites were mutated, and HOTAIR-mutant (MUT) and PTEN-MUT were constructed. After that, HOTAIR-WT, HOTAIR-MUT, PTEN$\mathrm{WT}$, and PTEN-MUT plasmids were mixed and cotransfected with miR-NC and miR-148a mimic plasmids, respectively, into $293 \mathrm{~T}$ cells (American Type Culture Collection, Manassas, Virginia, USA). After $48 \mathrm{~h}$ transfection, the cells were collected and lysed. The luciferase activity was determined using a luciferase detection kit (BioVision, San Francisco, CA, USA) and GloMax 20/20 luminometer (Promega, Madison, WI, USA).

2.15. RNA Pull-Down Assay. An Eppendorf (EP) tube without RNase was added with $50 \mu \mathrm{L}$ magnetic beads, $20 \mathrm{mM}$ Tris solution ( $\mathrm{pH}=7.5), 1 \times$ RNA capture buffer, and 50 pmol biotin-labeled miR-143, respectively, for $2 \mathrm{~h}$ incubation at $37^{\circ} \mathrm{C}$. Afterwards, the incubated EP tube was added with $2 \mu \mathrm{L}$ DNase I for $15 \mathrm{~min}$ incubation at $37^{\circ} \mathrm{C}$. The reaction was terminated by the addition of $2 \mu \mathrm{L}$ ethylenediaminetetraacetic acid (EDTA) $(0.2 \mathrm{M}, \mathrm{pH}=8.0)$. Biotin-labeled RNA $(1 \mu \mathrm{g})$ was added with a proper amount of structure buffer $(10 \mathrm{mM}$ Tris, $\mathrm{pH}=7.0 ; 0.1 \mathrm{M} \mathrm{KCl} ; 10 \mathrm{mM}$ $\mathrm{MgCl}_{2}$ ), heated at $95^{\circ} \mathrm{C}$ for $2 \mathrm{~min}$, put on the ice bath for $3 \mathrm{~min}$, and rested at room temperature for $30 \mathrm{~min}$. Afterwards, the magnetic bead-RNA mixture was added with cell lysis buffer (containing about $1 \mathrm{mg}$ protein). An appropriate amount of RNase inhibitor was added to the lysis buffer, which was then placed at room temperature for $1 \mathrm{~h}$ and centrifuged at low speed with the supernatant recovered as the NC of the system, followed by 3 washes with Wash Buffer II (1 $\mathrm{mL}$ per wash). The liquid was added or dripped along the wall, turned up and down slowly, and mixed thoroughly. Blowing using pipetting was not allowed. Finally, the RNA product was obtained, and HOTAIR expression was detected using RT-qPCR.

2.16. Animal Treatment. A total of 32 adult male SpragueDawley rats (230-260 g, 7-10 weeks) (Shanghai Branch of Beijing Vital River Laboratory Animal Technology Co., Ltd. (Beijing, China); SYXK (Shanghai) 2017-0014)) were kept in the standard animal room at $18-22^{\circ} \mathrm{C}$ and provided with freely available food and water under a light/dark cycle. All animals were euthanized by intraperitoneal injection of pentobarbital sodium $(\geq 100 \mathrm{mg} / \mathrm{kg})$.

2.17. Establishment of IDD Animal Model. A total of 32 rats were randomly allocated to the sham group, IDD + PBS group, IDD + si-NC group, and IDD + si-HOTAIR group, with 8 rats in each group. All the animals were anesthetized using $40 \mathrm{mg} / \mathrm{kg}$ pentobarbital sodium ( $2 \%(\mathrm{w} / \mathrm{v}))$. The IVDs (Co6-7, Co7-8, and Co8-9) in the tailbone of the rats that needed IDD modeling were located by palpation and then confirmed using radiography. The AF (Co6-7 and Co8-9, about $4 \mathrm{~mm}$ deep) was punctured using a needle $(27 \mathrm{G})$ through the caudal skin, parallel to the endplates. Next, all needles were rotated $360^{\circ}$ axially and placed for $1 \mathrm{~min}$ [10]. Subsequently, $2 \mu \mathrm{L}$ PBS, si-NC, or si-HOTAIR was slowly injected into the target tailbone, and the needle was kept for $2 \mathrm{~min}$. All animals were allowed free loading and movement. All rats were euthanized 4 weeks after IDD surgery. Caudal IVDs and adjacent vertebral bodies were collected from 4 
rats for histological evaluation. The IVD tissues of the remaining 4 rats were homogenized for subsequent $\mathrm{WB}$ and RT-qPCR experimentations.

2.18. Histological Evaluation. Four weeks after IDD operation, the rats were euthanized, and the IVDs and adjacent vertebral bodies were resected and fixed with $10 \%$ formalin. The samples were decalcified in 10\% EDTA for 30 days, embedded in paraffin, and then sectioned $(5 \mu \mathrm{m})$ using a microtome (Leica RM 2145, Leica Microsystems, Nussloch, Germany). Hematoxylin and eosin (HE) and TUNEL staining were used for histological evaluation. The histological evaluation was conducted employing previously published methods [24].

2.19. Statistical Analysis. Data were analyzed utilizing SPSS 21.0 (IBM Corp. Armonk, NY, USA). Kolmogorov-Smirnov test verified that data were in the normal distribution. The results were represented as mean \pm standard deviation. The independent sample $t$-test was utilized for comparative analysis between two groups, and one-way analysis of variance (ANOVA) was utilized for comparative analysis among multiple groups followed by Tukey's multiple comparisons test. $p<0.05$ indicated statistically significant difference; $p<0.01$ represented highly statistically significant difference.

\section{Results}

3.1. HOTAIR Was Highly Expressed in NP Cells of IDD Patients. LncRNAs are critical in IDD pathogenesis [11]. To study the role of lncRNA HOTAIR in NP cells, we first isolated and cultured normal and degenerative NP cells from NP tissue samples obtained from clinical disc surgery. NP cells of P1, P2, and P3 generations were compared. NP cells of P2 generation with full morphology, abundant cytoplasm, strong refraction and metabolism, and the strongest proliferation ability were selected for subsequent experiments (Figure 1(a)). ICC staining of Collagen II showed that the cytoplasm of normal NP cells was stained with typical brown yellow, and as it approached the nucleus, the staining became darker, while the cytoplasmic staining of degenerative NP cells was lighter than that of normal NP cells, showing light yellow (Figure 1(b)). Similar results were also found in toluidine blue staining, which showed blue color in normal $\mathrm{NP}$ cell cytoplasm and light blue color in degenerative NP cell cytoplasm (Figure 1(b)), together with reduced aggrecan generation compared with normal NP cells. To further confirm the results of ICC and toluidine blue staining, we detected Collagen II and aggrecan mRNA expression in normal and degenerative NP cells of P2 generation using RT-qPCR. Collagen II and aggrecan mRNA expressions were remarkably reduced in degenerative NP cells relative to those in normal NP cells (both $p<0.05$ ) (Figure 1(c)). Normal and degenerative NP cell proliferation was determined using MTT assay, which demonstrated increased OD value of the two groups with the extension of culture time and lower OD values in degenerative NP cells than those in normal NP cells on days 2-4 $(p<0.05)$ (Figure 1(d)), indicating the lower proliferation ability than normal NP cells. The above results were consistent with the growth characteristics of degenerative NP cells. Finally, HOTAIR expression in normal and degenerative NP cells was detected by RT-qPCR, which found remarkably elevated HOTAIR expression in degenerative NP cells $(p<0.01)$ (Figure 1(e)).

3.2. HOTAIR Knockdown Inhibited Autophagy and Apoptosis of Degenerative NP Cells. To study the specific role of HOTAIR in autophagy and apoptosis of NP cells, we reduced HOTAIR expression by transfecting si-HOTAIR into degenerative NP cells, and the successful transfection was verified using RT-qPCR $(p<0.05)$ (Figure $2(\mathrm{a})$ ). Levels of autophagy-related proteins (LC3II/I, p62, and Beclin-1) were then detected using WB. si-HOTAIR treatment reduced LC3II/I and Beclin-1 levels and elevated p62 levels (all $p<0.05$ ) (Figure 2(b)). According to MDC fluorescence staining results, degenerative NP cells transfected with siHOTAIR exhibited markedly decreased autophagic cells $(p<0.05)$ (Figure $2(c))$, suggesting inhibition of degenerative NP cell autophagy by HOTAIR knockdown.

As shown by TUNEL staining and flow cytometry results, si-HOTAIR-treated degenerative NP cells showed reduced apoptosis rates (all $p<0.05$ ) (Figures $2(\mathrm{~d})$ and $2(\mathrm{e})$ ). These results indicated that silencing HOTAIR impeded autophagy and apoptosis of degenerative NP cells.

3.3. HOTAIR Acting as a ceRNA Competitively Bound to miR-148a to Upregulate PTEN Expression. The action mechanism of lncRNA depends on its location in cells. To explore the mechanism of HOTAIR in autophagy and apoptosis of NP cells, we first predicted lncRNA HOTAIR location through the database (https://www.csbio.sjtu.edu. $\mathrm{cn} /$ bioinf/lncLocator/) [25]. HOTAIR was mainly located in the cytoplasm (Figure 3(a)). Next, HOTAIR subcellular localization in NP cells was analyzed using RNA-FISH and nuclear and cytoplasmic fractionation assays, which verified that HOTAIR was mainly located in NP cell cytoplasm (both $p<0.01$ ) (Figures 3(b) and 3(c)). The above findings suggested that HOTAIR may regulate NP cells through the mechanism of ceRNA.

Subsequently, we predicted that many miRNAs can bind to HOTAIR through the database (https://starbase.sysu.edu. cn/agoClipRNA.php?source=lncRNA) [26], among which miR-148a is common in IDD tissues and related to the IDD mechanism [27]. Therefore, we speculated that miR-148a may be a key miR in the ceRNA network of HOTAIR. Next, based on the binding sites of HOTAIR and miR-148a (Figure 3(d)), the binding relationship between the two was verified using dual-luciferase assay and RNA pull-down (all $p<0.05$ ) (Figures 3(e) and 3(f)). Finally, miR-148a expression in degenerative NP cells transfected with siHOTAIR was determined using RT-qPCR, which showed a negative correlation with HOTAIR expression $(p<0.05)$ (Figure 3(g)).

Starbase database (https://starbase.sysu.edu.cn/ agoClipRNA.php?source=lncRNA) predicted that 
P1

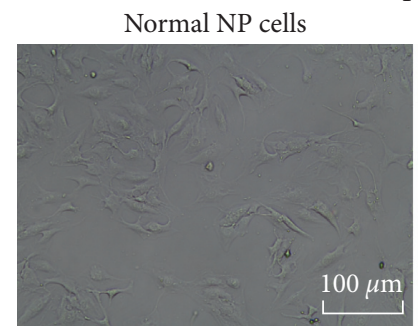

Degenerative NP cells

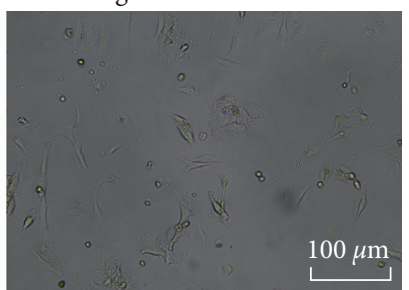

P2

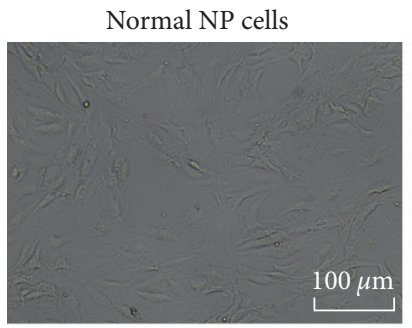

Degenerative NP cells

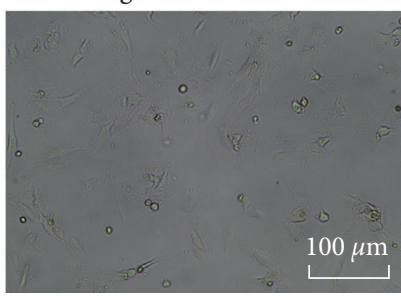

P3

Degenerative NP cells

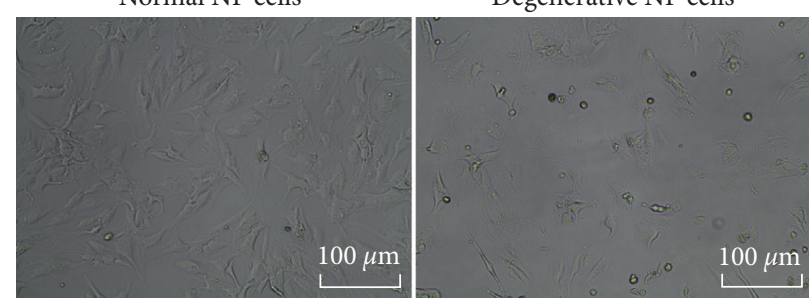

(a)

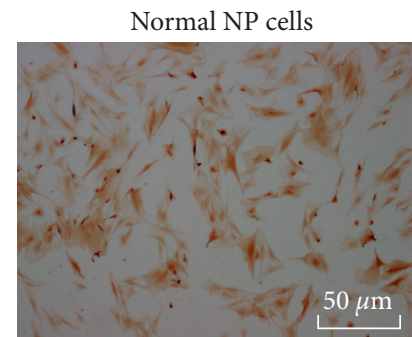

Degenerative NP cells
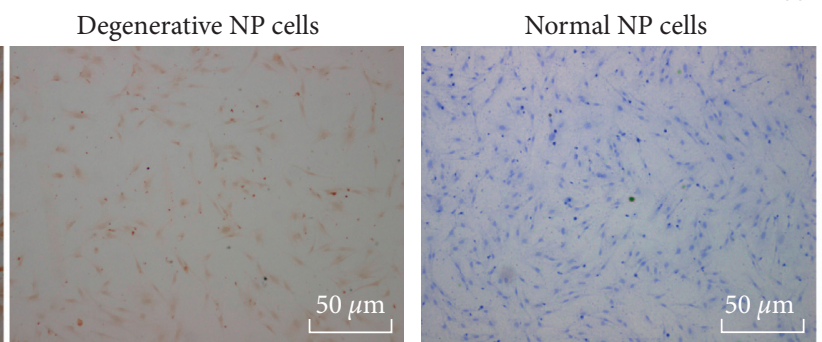

Degenerative NP cells

(b)

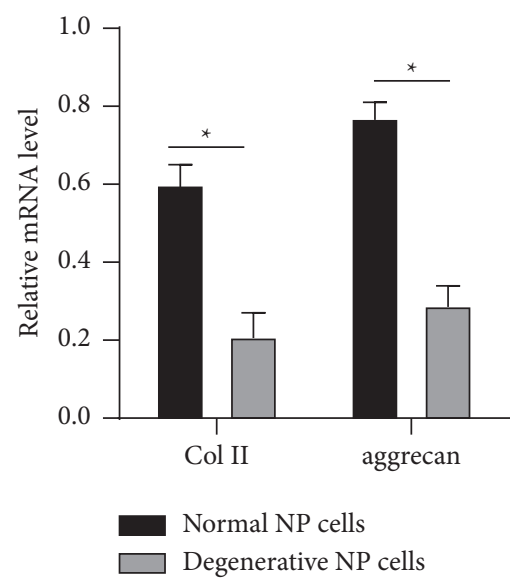

(c)

Figure 1: Continued. 


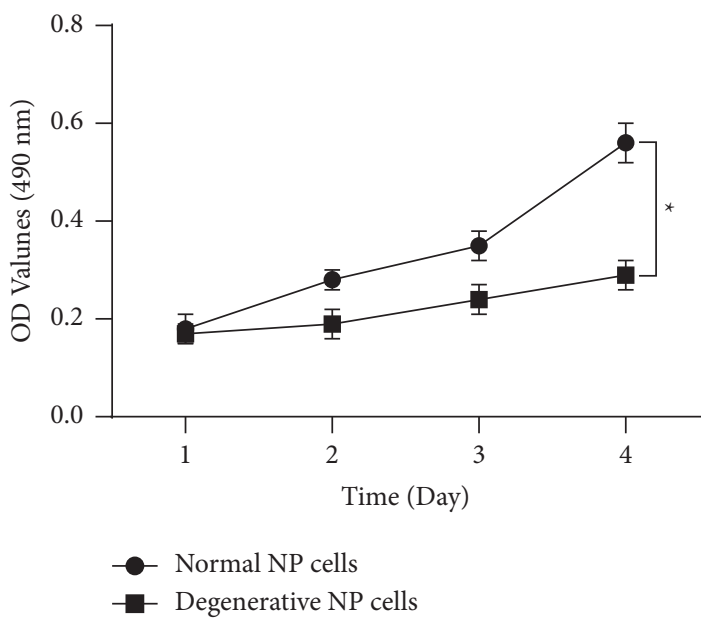

(d)

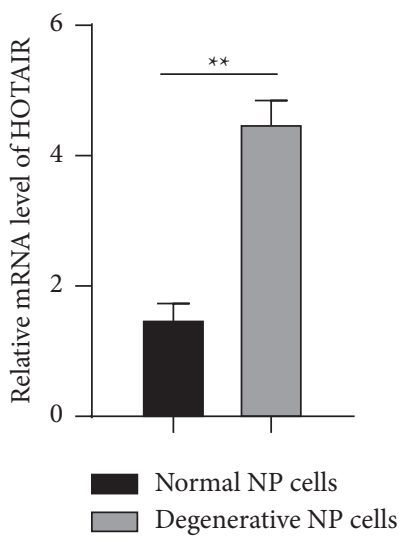

(e)

FIGURE 1: HOTAIR is highly expressed in NP cells of IDD patients. (a) The morphological changes of normal and degenerative NP cells were observed using an inverted phase-contrast microscope. (b) Normal and degenerative NP cells were observed by ICC and toluidine blue staining. (c) Collagen II and aggrecan mRNA expressions in normal and degenerative NP cells were detected using RT-qPCR. (d) MTT assay was used to detect the proliferation of normal and degenerative NP cells of P2 generation. (e) RT-qPCR was used to detect the expression of HOTAIR in normal and degenerative NP cells. The experiment was repeated three times, and the data were expressed as mean \pm standard deviation. Data comparisons between two groups were analyzed using independent $t$-test. ${ }^{*} p<0.05 ;{ }^{* *} p<0.01$.

miR-148a has targeting relationships with multiple genes, among which PTEN is associated with IDD [28]. Therefore, according to the binding sites of miR-148a and PTEN $3{ }^{\prime} U T R$ (Figure 3(h)), we verified that miR-148a targeted PTEN using a dual-luciferase assay $(p<0.05)$ (Figure 3(i)). In addition, miR-148a mimic or miR-148a inhibitor was transfected into degenerative NP cells, which markedly increased or decreased miR-148a expression, correspondingly (both $p<0.05$ ) (Figure $3(j)$ ), along with notably reduced or elevated mRNA expression of PTEN. Moreover, PTEN mRNA expression was dramatically reduced after siHOTAIR treatment (all $p<0.05$ ) (Figure $3(\mathrm{k})$ ). From all above, HOTAIR functioning as a ceRNA competitively bound to miR-148a and upregulated PTEN mRNA expression.

3.4. miR-148a Knockdown Reversed the Inhibition of si-HOTAIR on Degenerative NP Cell Autophagy and Apoptosis.
Firstly, miR-148a expression in normal and degenerative NP cells was measured. According to RT-qPCR results, miR-148a expression in degenerative NP cells was remarkably reduced relative to that in normal NP cells $(p<0.05)$ (Figure 4(a)). Next, combined experiments were conducted to confirm the above ceRNA network. miR-148a inhibitor/inhibitor NC was transfected into siHOTAIR-treated degenerative NP cells (si-HOTAIR + inNC group/si-HOTAIR + in-miR-148a group). Levels of autophagy-related proteins (p62, LC3II/I, and Beclin-1) were detected using $\mathrm{WB}$, which showed diminished p62 levels, and augmented LC3II/I and Beclin-1 levels after siHOTAIR + in-miR-148a treatment, indicating that miR148a knockdown reversed the effect of si-HOTAIR on autophagy of degenerative NP cells (all $p<0.05$ ) (Figure 4(b)). According to flow cytometry results, degenerative NP cells exhibited noticeably elevated apoptosis rate after si-HOTAIR + in-miR-148a treatment $(p<0.05)$ (Figure 4(c)). Collectively, silencing miR-148a 


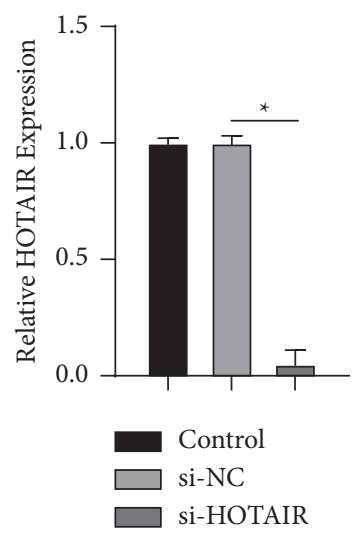

(a)
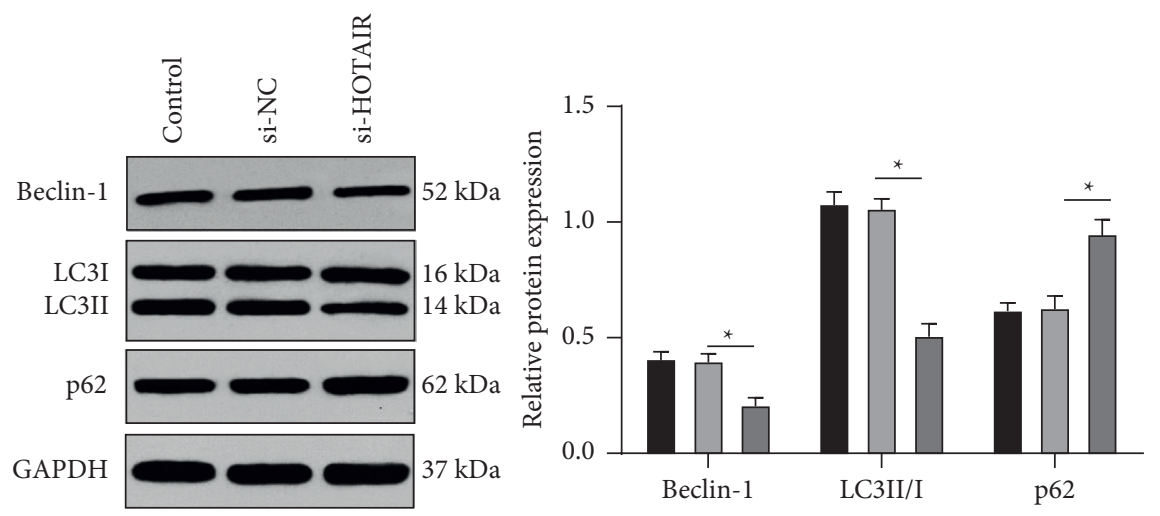

Control

$\square$ si-NC

$\square$ si-HOTAIR

(b)

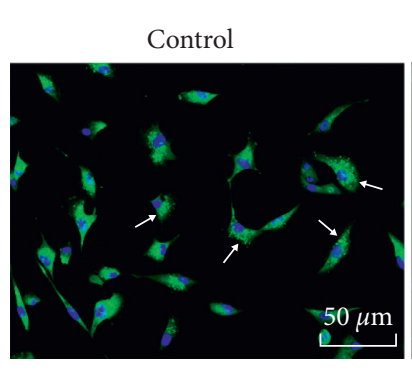

si-NC

si-HOTAIR
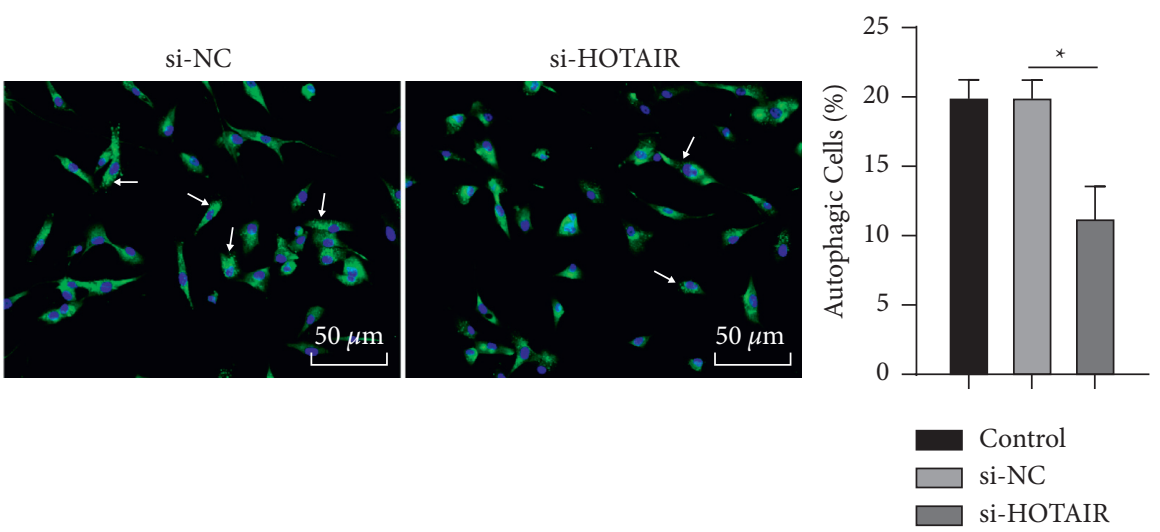

(c)

Figure 2: Continued. 

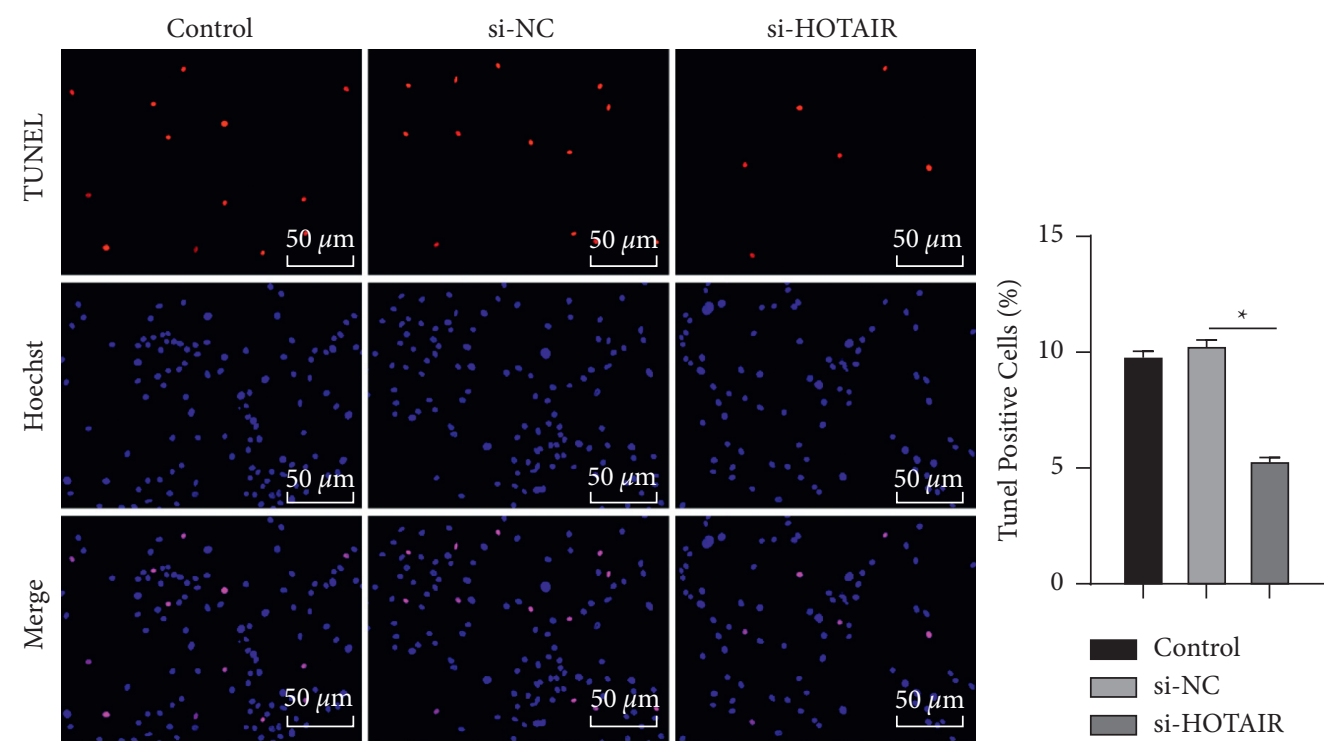

(d)
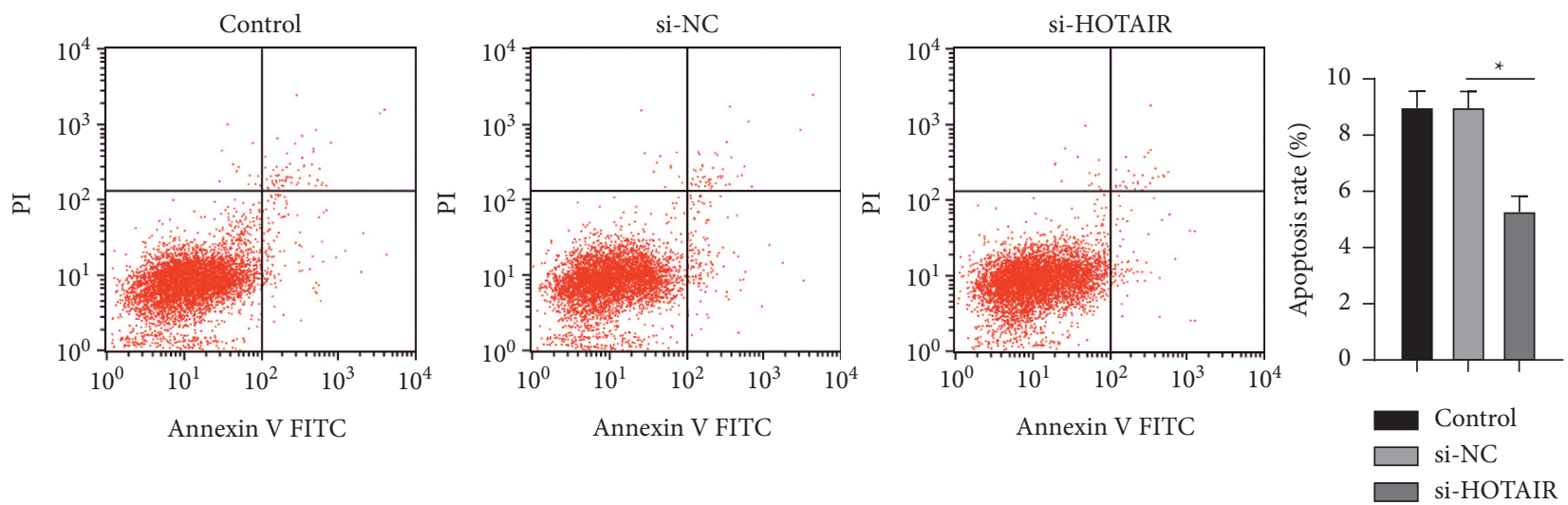

(e)

FIgURE 2: HOTAIR knockdown inhibits autophagy and apoptosis of degenerative NP cells. si-HOTAIR was transfected into degenerative NP cells. (a) The expression of HOTAIR was detected using RT-qPCR. (b) Levels of autophagy-related proteins (LC3II/I, p62, and Beclin-1) were detected using WB. (c) The number of autophagic cells was detected using MDC staining. (d, e) The apoptosis of degenerative NP cells in each group was detected using TUNEL staining and flow cytometry. The experiment was repeated three times, and the data were expressed as mean \pm standard deviation. Data comparisons among multiple groups were analyzed using one-way ANOVA, followed by Tukey's multiple comparisons test. ${ }^{*} p<0.05$.

reversed the impact of HOTAIR on degenerative NP cell autophagy and apoptosis.

\subsection{Silencing HOTAIR Inhibited Autophagy and Apoptosis} and Improved Pathological Damage of NP Cells in IDD Rats. Our in vitro experiments confirmed that HOTAIR knockdown inhibited degenerative NP cell autophagy and apoptosis, which was further verified in vivo. IDD rats were firstly induced, and we found that HOTAIR expression in NP cells of IDD rats was notably increased and then successfully reduced after siHOTAIR treatment (all $p<0.05$ ) (Figure 5(a)). The pathological condition of NP tissues in each group was evaluated using HE staining and scored by histological evaluation. IDD rats exhibited remarkable NP degeneration and notably reduced histological score compared with sham-operated rats, while attenuated NP degeneration and an increased histological score were observed after si-HOTAIR treatment $(p<0.05)$ (Figure 5(b)). The above results indicated that silencing HOTAIR improved pathological damage of IDD rats.

Next, levels of autophagy-related proteins (p62, LC3II/I, and Beclin-1) in rat IVD tissues were detected. As shown by WB results, si-HOTAIR-treated IDD rats exhibited notably reduced LC3II/I and Beclin-1 levels and increased p62 levels, which indicated the attenuation of autophagy in IDD rats by si-HOTAIR (all $p<0.05$ ) (Figure 5(c)). TUNEL staining revealed that the apoptosis rate of IDD rats was reduced after si-HOTAIR treatment $(p<0.05)$ (Figure 5(e)). Furthermore, as indicated by RT-qPCR results, compared with the IDD + si-NC group, the IDD + si-HOTAIR group showed markedly elevated miR-148a expression $(p<0.05)$ (Figure 5(d)) and notably reduced PTEN mRNA expression $(p<0.05)$ (Figure 5(f)). Overall, the above in vitro and in vivo experiment results revealed that HOTAIR knockdown 


….. IncLocator Prediction Result $\ldots \ldots .$.
\begin{tabular}{|c|c|}
\hline Subcellular locations & score \\
\hline Cytoplasm & 0.476097823965 \\
\hline Nucleus & 0.308459398811 \\
\hline Ribosome & 0.0486406897103 \\
\hline Cytosol & 0.0264526073091 \\
\hline Exosome & 0.140349480205 \\
\hline & Predicted location \\
\hline \multicolumn{2}{|c|}{ Cytoplasm } \\
\hline
\end{tabular}

(a)

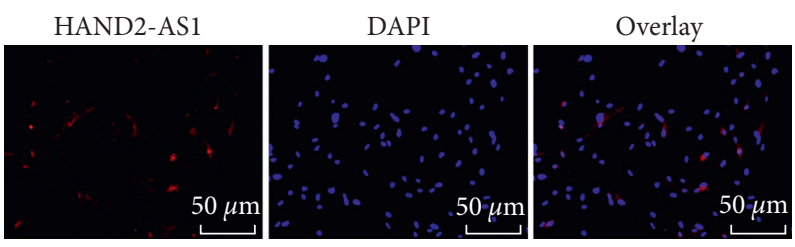

(b)

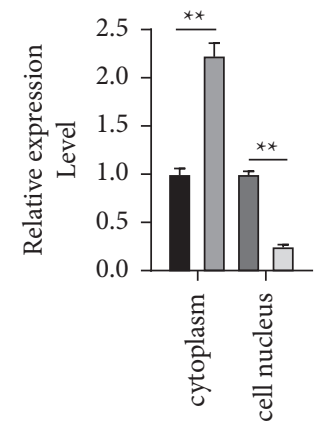

GAPDH

$\square$ IncRNA HOTAIR

$\square$ U6

$\square$ IncRNA HOTAIR

(c)

\begin{tabular}{|c|c|c|c|c|c|c|}
\hline miRNA & GeneID & GeneName & GeneType & TargetSite & $\uparrow \downarrow$ & Alignment \\
\hline hsa-miR-148a-3p $\uparrow$ & ENSG00000247556 & OIP5-AS1 $\uparrow$ & processed_transcript & chr15:41598708-41598730[+] & $\uparrow$ & $\begin{array}{c}\text { Target: } 5 \text { ' agcAUGGGCUGAGAAUGCACUGa } 3^{\prime} \\
\text { | । III । IIIIIII } \\
\text { miRNA : } 3^{\prime} \text { uguUUCAAGAC-AUCACGUGACu } 5^{\prime}\end{array}$ \\
\hline
\end{tabular}

(d)

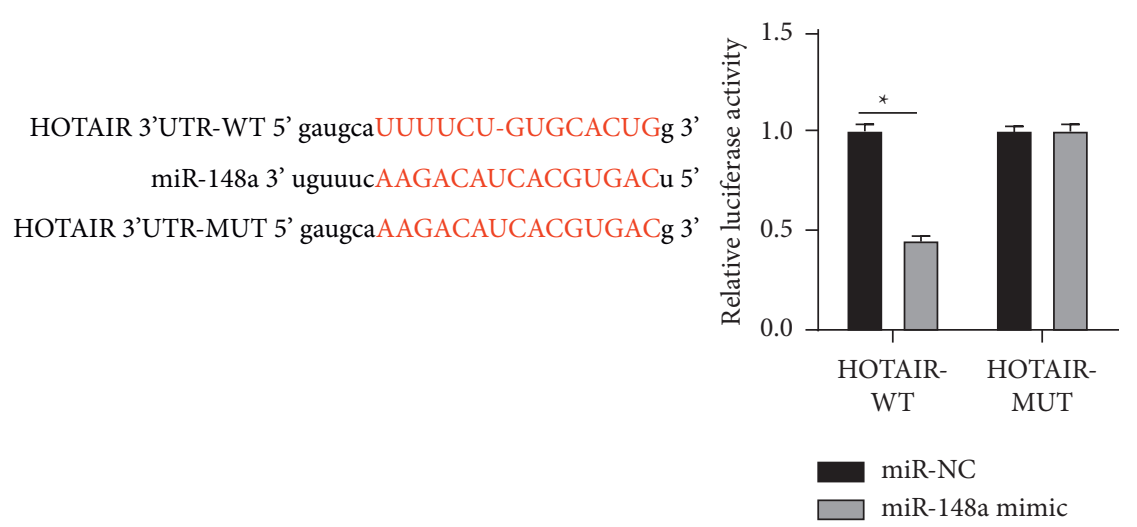

(e)

Figure 3: Continued. 


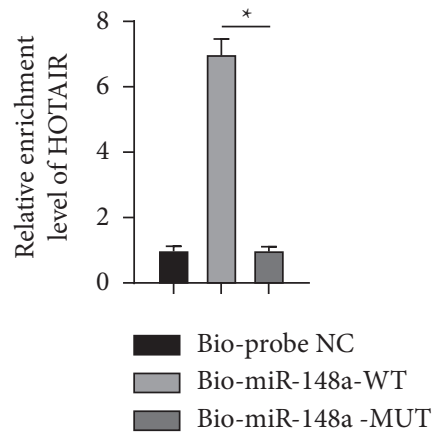

(f)

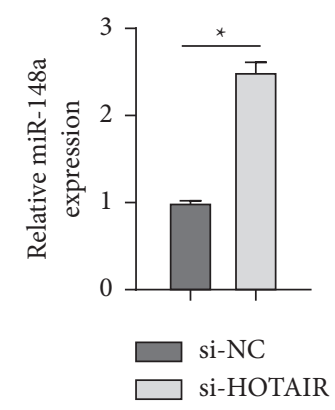

(g)

\begin{tabular}{|c|c|c|c|}
\hline BindingSite & $\uparrow$ & Class & Alignment \\
\hline chr10:89727483-89727489[+] & $\uparrow$ & 7 mer-m8 8 & 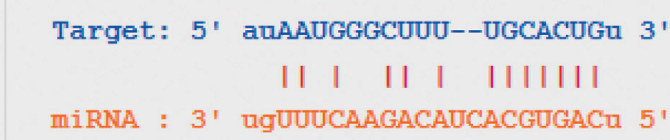 \\
\hline
\end{tabular}

(h)

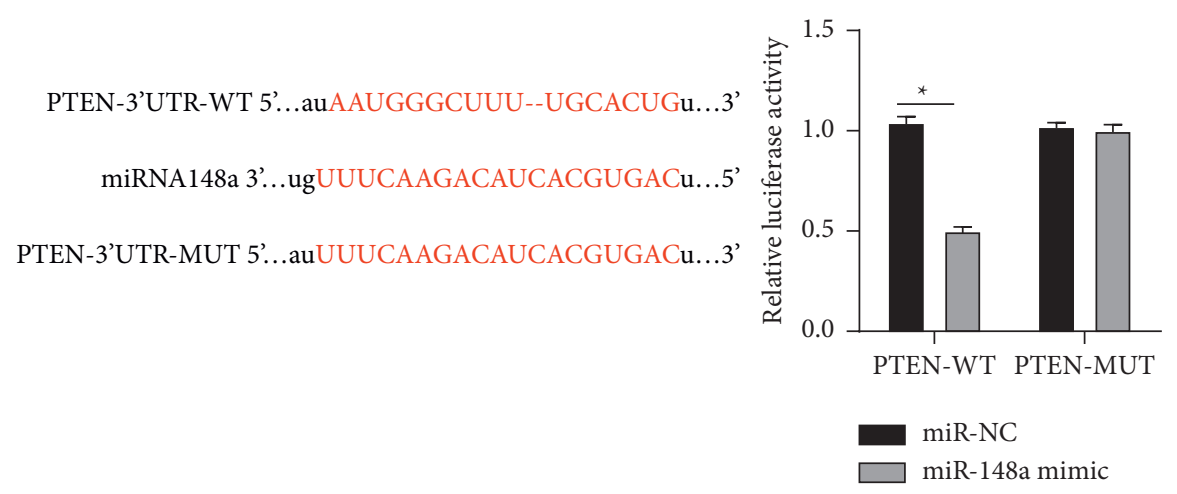

(i)

Figure 3: Continued. 


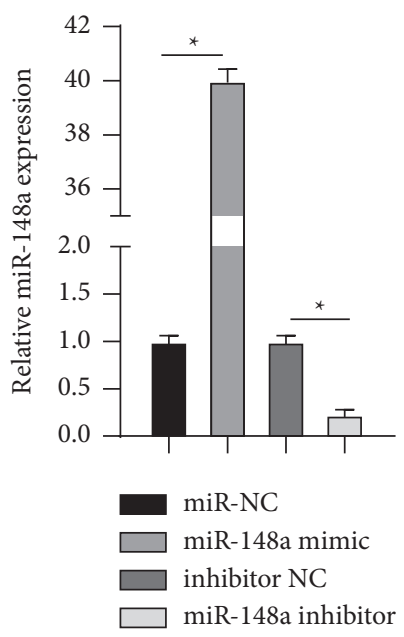

(j)

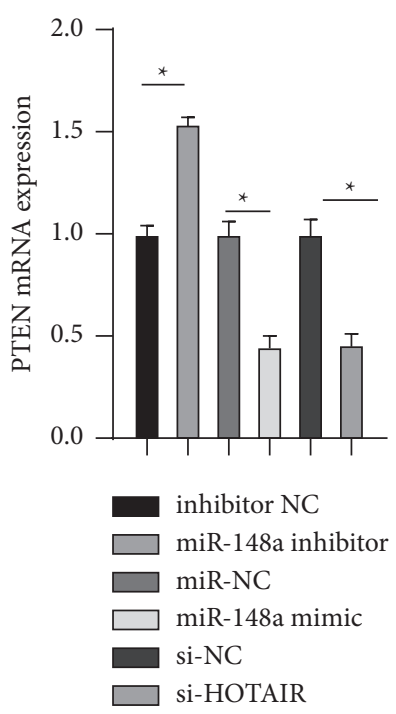

(k)

FIgURE 3: HOTAIR acting as a ceRNA competitively binds to miR-148a to upregulate PTEN mRNA expression. (a) The lncRNA HOTAIR location was predicted through the database (https://www.csbio.sjtu.edu.cn/bioinf/lncLocator/). (b) The fluorescence localization of HOTAIR in NP cells was detected using RNA-FISH. (c) The expression of HOTAIR was detected using RT-qPCR after nuclear and cytoplasmic fractionation assay. (d) The binding sites of HOTAIR and miR-148a were predicted through the bioinformatics website (https:// starbase.sysu.edu.cn/agoClipRNA.php?source=lncRNA). (e) Dual-luciferase reporter gene assay was used to verify the binding relationship between HOTAIR and miR-148a. (f) RNA pull-down was used to verify the binding relationship between HOTAIR and miR-148a in NP cells. (g) RT-qPCR was used to detect miR-148a expression after HOTAIR knockdown. (h) Bioinformatics website (http://starbase.sysu.edu. cn/agoClipRNA.php?source=lncRNA) was used to predict the binding sites of miR-148a and PTEN. (i) Dual-luciferase reporter gene assay was used to verify the binding relationship between miR-148a and PTEN. (j) RT-qPCR was used to detect the expression of miR-148a. (k) RT-qPCR was used to detect PTEN mRNA expression. The experiment was repeated three times, and the data were expressed as mean \pm standard deviation. Data in panels $\mathrm{E} / \mathrm{G} / \mathrm{I}$ were analyzed using $t$-test; data in panels $\mathrm{C} / \mathrm{F} / \mathrm{J} / \mathrm{K}$ were analyzed using one-way ANOVA, followed by Tukey's multiple comparisons test. ${ }^{*} p<0.05 ;{ }^{* *} p<0.01$.

elevated miR-148a expression to downregulate PTEN expression, thereby inhibiting degenerative NP cell autophagy and apoptosis and improving IDD.

\section{Discussion}

IDD contributes largely to low back pain and even disability [6]. LncRNAs are intrinsically related to NP cell functions in IDD [8]. This study demonstrated that lncRNA HOTAIR functioned as a ceRNA of miR-148a to promote PTEN mRNA expression, thereby inducing NP cell autophagy and apoptosis in IDD.

LncRNAs are implicated in IDD pathogenesis [7]. Deregulated NP cells participate in IDD initiation and progression and can be regulated by lncRNAs in IDD [8]. A previous study has pointed out that IncRNA HOTAIR plays a regulatory role in NP cells in IDD [11]. According to our results, NP cells in IDD patients exhibited degenerative 


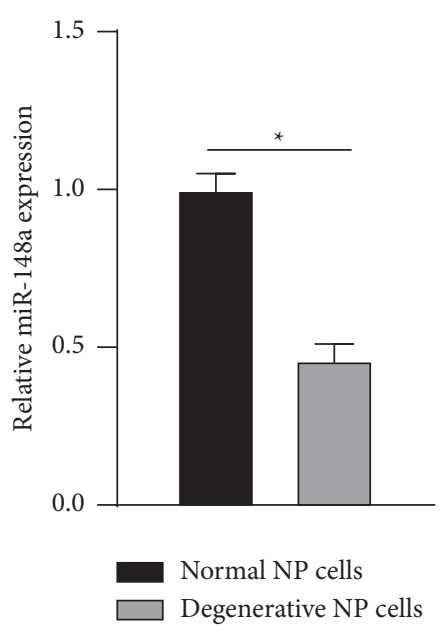

(a)

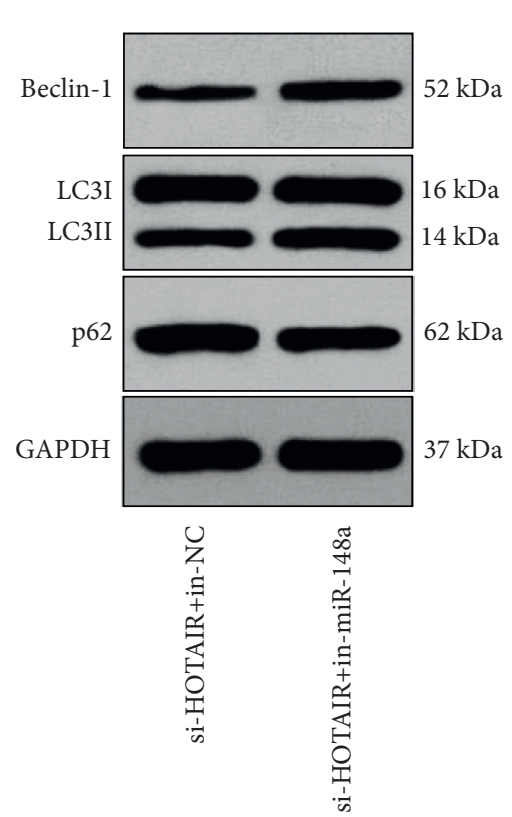

(b)

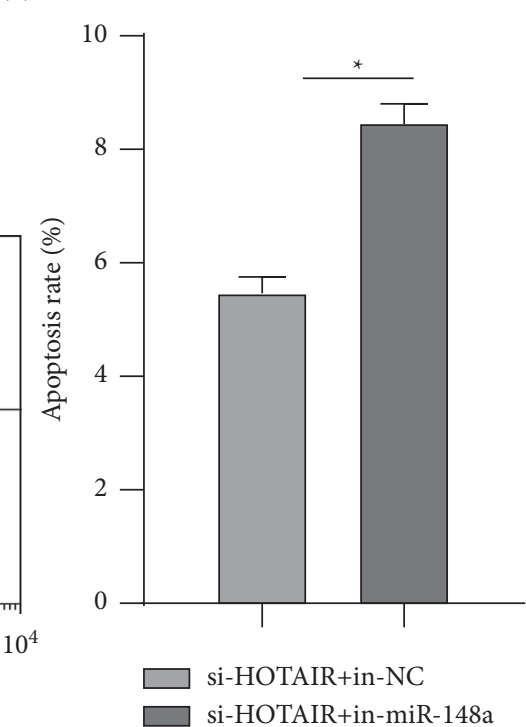

(c)

FIGURE 4: miR-148a knockdown reverses the inhibition of si-HOTAIR on the autophagy and apoptosis of degenerative NP cells. miR-148a inhibitor/inhibitor NC was transfected into degenerative NP cells with si-HOTAIR treatment group (si-HOTAIR + in-NC group/siHOTAIR + in-miR-148a group). (a) The expression of miR-148a in normal and degenerative NP cells was detected using RT-qPCR. (b) Levels of autophagy-related proteins (p62, LC3II/I, and Beclin-1) were detected using WB. (c) Apoptosis was detected using flow cytometry. The cell experiment was repeated three times, and the data were expressed as mean \pm standard deviation. Data comparisons between two groups were analyzed using $t$-test. ${ }^{*} p<0.05$.

characteristics, and HOTAIR expression was remarkably elevated in degenerative NP cells. Consistently, HOTAIR expression in NP cells shows a positive correlation with IDD grade [10]. Thereafter, we silenced HOTAIR expression in degenerative NP cells to further verify the effects of HOTAIR in IDD. It is well established that the imbalance between autophagy and apoptosis of NP cells makes great contributions to IDD progression [2, 29]. As presented by our findings, si-HOTAIR treatment reduced LC3II/I and Beclin1 levels and elevated p62 levels in degenerative NP cells, accompanied by dramatically reduced apoptosis rate. In previous studies, increased LC3II/I and Beclin-1 levels and reduced p62 levels in NP cells are strong indicators of autophagy $[30,31]$. In agreement with these, HOTAIR knockdown attenuates the degeneration process and IDD symptoms and inhibits NP cell apoptosis [11]. Our in vivo experiments further verified that silencing HOTAIR inhibited degenerative NP cell autophagy and apoptosis in IDD rats, with manifestations of noticeably attenuated NP cell autophagy and apoptosis and markedly improved 


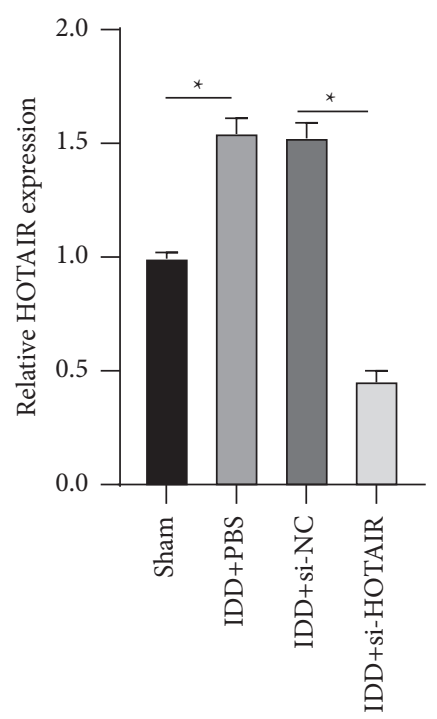

(a)

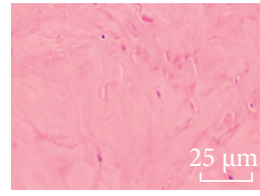

Sham

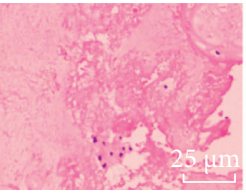

IDD+PBS

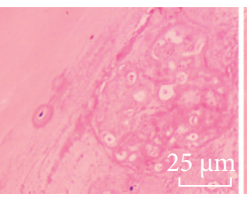

$\mathrm{IDD}+\mathrm{si}-\mathrm{NC}$

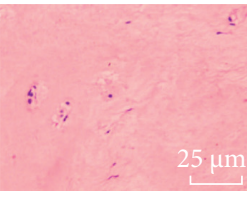

IDD+si-HOTAIR

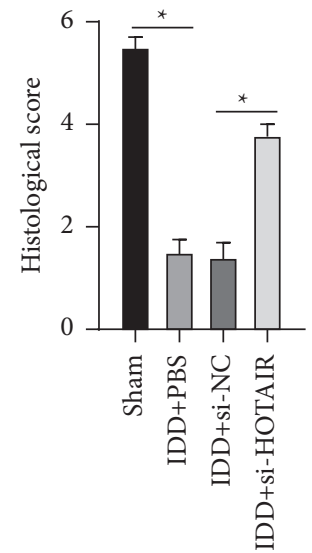

(b)
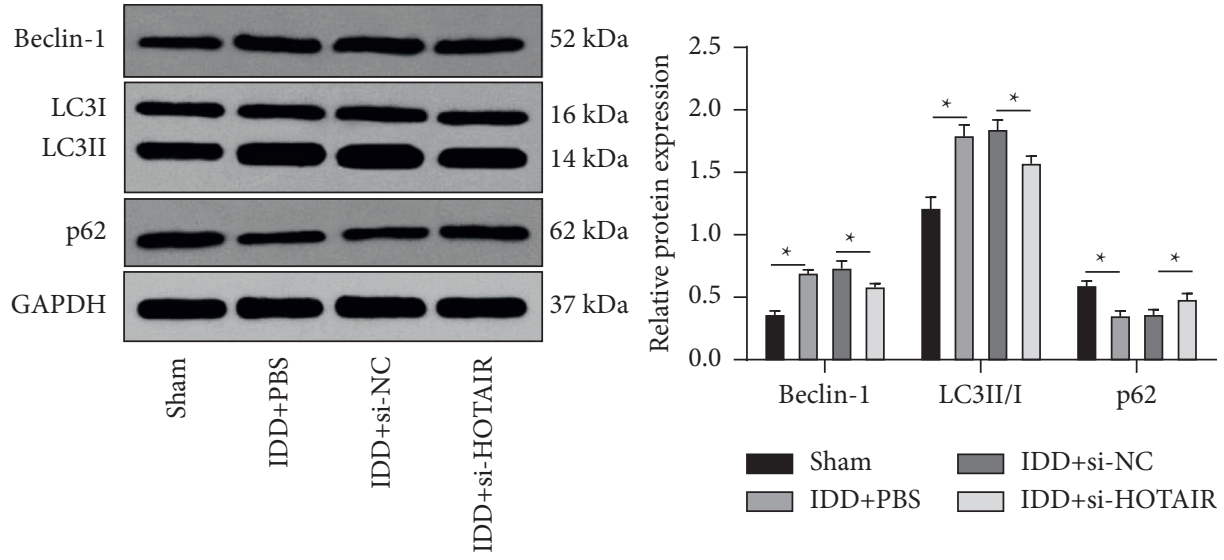

(c)

Figure 5: Continued. 


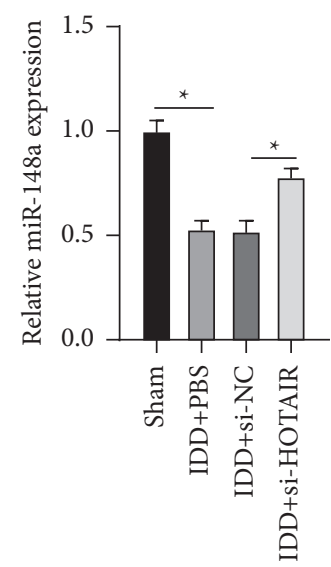

(d)
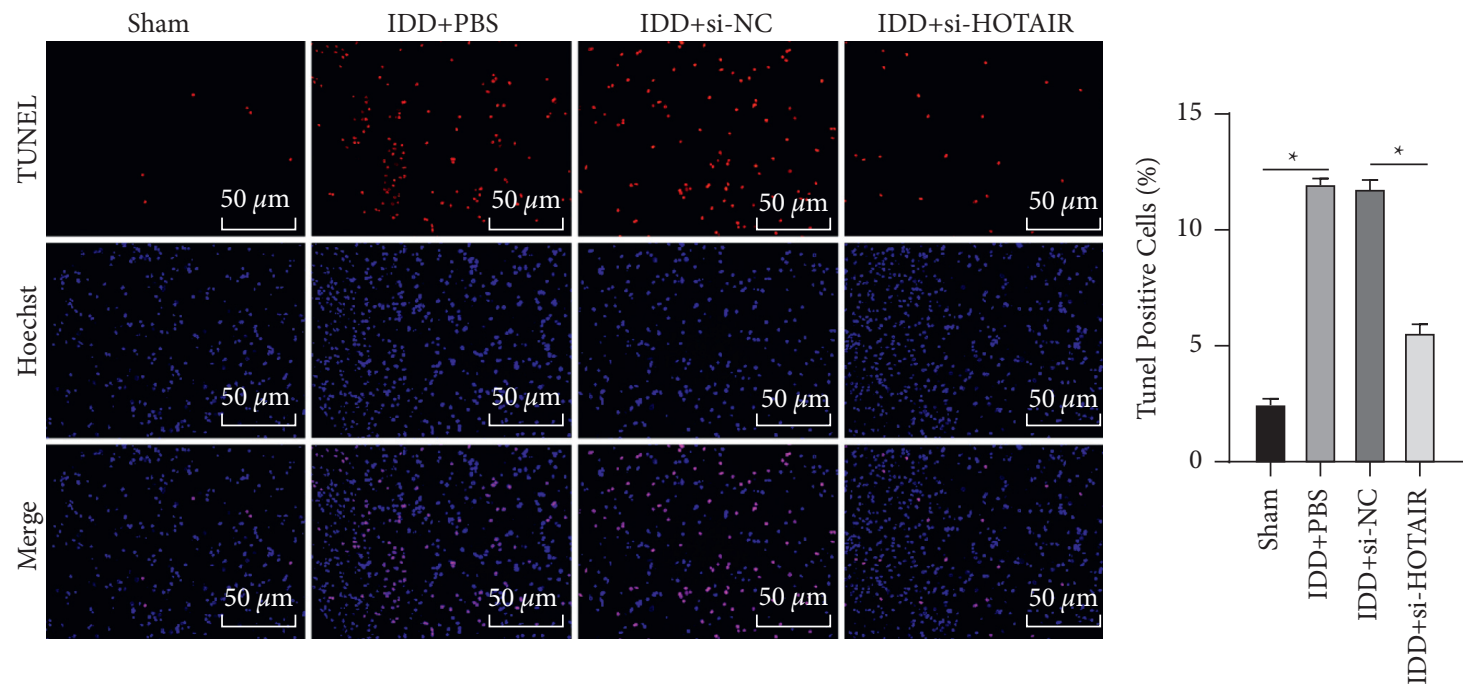

(e)

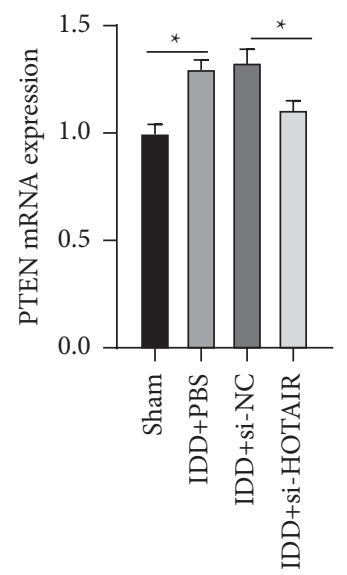

(f)

FIGURE 5: Silencing HOTAIR inhibits autophagy and apoptosis and improves pathological damage of NP cells in IDD rats. (a) RT-qPCR was used to detect the expression of HOTAIR in IVD tissues. (b) The pathological condition of NP tissues in each group was evaluated using HE staining and scored by histological evaluation. (c) WB was used to detect the levels of autophagy-related proteins (p62, LC3II/I, and Beclin-1) in IVD tissues. (d). RT-qPCR was used to detect the expression of miR-148a in IVD tissues. (e) TUNEL staining was used to detect apoptosis. (f) RT-qPCR was used to detect PTEN mRNA expression in IVD tissues. The experiment was repeated three times, and the data were expressed as mean \pm standard deviation. Data comparisons among multigroups were analyzed using one-way ANOVA followed by Tukey's multiple comparisons test. ${ }^{*} p<0.05$. 
pathological damage in IDD rats. Likewise, in a prior study, HOTAIR has been proven to stimulate autophagy and promote apoptosis of NP cells, thereby aggravating IDD [10]. Overall, we highlighted that silencing HOTAIR impeded autophagy and apoptosis of degenerative NP cells.

As has been pointed out previously, lncRNAs play a nonneglectable role in IDD via a lncRNA-miRNA-mRNA ceRNA network [13]. To explore the regulatory downstream mechanism of lncRNA HOTAIR, we predicted and identified miR-148a as a key miR in the ceRNA network of lncRNA HOTAIR in degenerative NP cells. Consistently, miR-148a shows a close relation with IDD progression [21]. Numerous studies have demonstrated that HOTAIR acts as a ceRNA of miR-148a in multiple diseases [32, 33]. Furthermore, we validated PTEN as a target gene for miR-148a in degenerative NP cells, and PTEN mRNA expression showed a positive correlation with HOTAIR expression and a negative correlation with miR-148a expression, which were further proven in IDD rats in vivo. Likewise, a previous study has proposed that PTEN is tightly involved in IDD development [34]. PTEN is overexpressed in degenerative NP cells and can induce NP cell apoptosis [28]. Accumulating studies documented the targeting relationship between miR148a and PTEN in diverse disorders [35, 36]. Additionally, HOTAIR can drive osteoarthritis progression, which is associated with the regulation of PTEN [25]. Briefly, we proved that HOTAIR acting as a ceRNA competitively bound to miR-148a to upregulate PTEN mRNA expression in degenerative NP cells.

Subsequently, we investigated the role of miR-148a in degenerative NP cells. We observed that miR-148a expression in degenerative NP cells was remarkably reduced, and miR-148a knockdown reversed the inhibitory effect of si-HOTAIR on the autophagy and apoptosis of degenerative NP cells. Likewise, in a prior study, miR-148a showed an aberrant downregulation in IDD [27]. miR-148-3p also protects against cell apoptosis in osteoarthritis [37]. Collectively, silencing miR-148a reversed the inhibition of siHOTAIR on degenerative NP cell autophagy and apoptosis.

All in all, this study supported that lncRNA HOTAIR knockdown suppressed NP cell autophagy and apoptosis via the miR-148a/PTEN axis in IDD. These results discovered a novel lncRNA-based therapy for IDD patients. However, HOTAIR was chosen as the research object after literature consulting, which is not the best strategy to screen key factors. It might be of more representative significance to screen other potential regulatory lncRNAs or HOTAIRmediated miRNAs in IDD development using the bioinformatics method (such as high throughput screening). This study simply revealed the role of the HOTAIR-regulated ceRNA network in NP cell autophagy and apoptosis in IDD, yet the clinical application of the HOTAIR/miR-148a/ PTEN axis in IDD needs to be further verified.

\section{Data Availability}

The data generated or analyzed during this study are included in this published article.

\section{Ethical Approval}

All the experimental protocols were recommended and got approval from the Ethics Committee of Wuxi 9th Affiliated Hospital of Soochow University (approval number: KT2019016). The experimental process strictly followed the approved protocol. Significant efforts were made to minimize animal numbers and suffering.

\section{Consent}

Consent from patients was obtained prior to sample collection.

\section{Disclosure}

The funding body did not participate in the design of the study and collection, analysis, and interpretation of data and in writing the manuscript.

\section{Conflicts of Interest}

The authors declare that they have no conflicts of interest.

\section{Authors' Contributions}

Shujun Zhang is the guarantor of the integrity of the entire study and contributed to the study concepts and study design. Sheng Song contributed to the definition of intellectual content and literature research. Wei Cui contributed to the manuscript preparation. Zhenzhong Sun contributed to the manuscript review. Shujun Zhang and Sheng Song contributed to the clinical studies, data acquisition, data analysis, and statistical analysis. Xueguang Liu and Wei Cui contributed to the experimental studies. All the authors read and approved the final manuscript.

\section{Acknowledgments}

This study was supported by the Natural Science Foundation of Hubei Province (no. 2018CFB713).

\section{References}

[1] F. Wang, F. Cai, R. Shi, X. H. Wang, and X. T. Wu, "Aging and age related stresses: a senescence mechanism of intervertebral disc degeneration," Osteoarthritis Cartilage, vol. 24, no. 3, pp. 398-408, 2016.

[2] F. Zhang, X. Zhao, H. Shen, and C. Zhang, "Molecular mechanisms of cell death in intervertebral disc degeneration (review)," International Journal of Molecular Medicine, vol. 37, no. 6, pp. 1439-1448, 2016.

[3] S. Chen, M. Luo, H. Kou, G. Shang, Y. Ji, and H. Liu, "A review of gene therapy delivery systems for intervertebral disc degeneration," Current Pharmaceutical Biotechnology, vol. 21, no. 3, pp. 194-205, 2020.

[4] K. Ma, S. Chen, Z. Li et al., "Mechanisms of endogenous repair failure during intervertebral disc degeneration," Osteoarthritis Cartilage, vol. 27, no. 1, pp. 41-48, 2019.

[5] C. Ruiz-Fernandez, V. Francisco, J. Pino et al., "Molecular relationships among obesity, inflammation and intervertebral 
disc degeneration: are adipokines the common link?" International Journal of Molecular Science, vol. 20, no. 8, 2019.

[6] P. Sampara, R. R. Banala, S. K. Vemuri, G. R. Av, and S. Gpv, "Understanding the molecular biology of intervertebral disc degeneration and potential gene therapy strategies for regeneration: a review," Gene Therapy, vol. 25, no. 2, pp. 67-82, 2018.

[7] W. K. Chen, X. H. Yu, W. Yang et al., "IncRNAs: novel players in intervertebral disc degeneration and osteoarthritis," Cell Proliferation, vol. 50, no. 1, 2017.

[8] Z. Li, X. Li, C. Chen et al., "Long non-coding RNAs in nucleus pulposus cell function and intervertebral disc degeneration," Cell Proliferation, vol. 51, no. 5, Article ID e12483, 2018.

[9] X. Wang, W. Liu, P. Wang, and S. Li, "RNA interference of long noncoding RNA HOTAIR suppresses autophagy and promotes apoptosis and sensitivity to cisplatin in oral squamous cell carcinoma," Journal of Oral Pathology Medicine, vol. 47, no. 10, pp. 930-937, 2018.

[10] S. Zhan, K. Wang, Q. Xiang et al., "IncRNA HOTAIR upregulates autophagy to promote apoptosis and senescence of nucleus pulposus cells," Journal of Cell Physiology, vol. 235, no. 3, pp. 2195-2208, 2020.

[11] S. Zhan, K. Wang, Y. Song et al., "Long non-coding RNA HOTAIR modulates intervertebral disc degenerative changes via Wnt/beta-catenin pathway," Arthritis Reserach and Therapy, vol. 21, no. 1, p. 201, 2019.

[12] Y. H. Zhang, J. Song, L. Shen, and J. Shao, "Systematic identification of lncRNAs and circRNAs-associated ceRNA networks in human lumbar disc degeneration," Biotechic Histochemistry, vol. 94, no. 8, pp. 606-616, 2019.

[13] J. Zhu, X. Zhang, W. Gao, H. Hu, X. Wang, and D. Hao, "lncRNA/circRNAmiRNAmRNA ceRNA network in lumbar intervertebral disc degeneration," Molecular Medicine Reports, vol. 20, no. 4, pp. 3160-3174, 2021.

[14] X. Jiang and D. Chen, "LncRNA FAM83H-AS1 maintains intervertebral disc tissue homeostasis and attenuates inflammation-related pain via promoting nucleus pulposus cell growth through miR-22-3p inhibition," Annals of Translational Medicine, vol. 8, no. 22, p. 1518, 2020.

[15] Z. Sun, X. Tang, H. Wang et al., "LncRNA H19 aggravates intervertebral disc degeneration by promoting the autophagy and apoptosis of nucleus pulposus cells through the miR-139/ CXCR4/NF-kappaB axis," Stem Cells Development, vol. 30, no. 14, pp. 736-748, 2021.

[16] J. Li, J. Chen, F. Zhang et al., "LncRNA CDKN2B-AS1 hinders the proliferation and facilitates apoptosis of ox-LDL-induced vascular smooth muscle cells via the ceRNA network of CDKN2B-AS1/miR-126-5p/PTPN7," International Journal of Cardiology, vol. 340, pp. 79-87, 2021.

[17] X. Zhou, L. Chen, S. Grad et al., "The roles and perspectives of microRNAs as biomarkers for intervertebral disc degeneration," Journal of Tissue Engineering and Regenerative Medicine, vol. 11, no. 12, pp. 3481-3487, 2017.

[18] J. Chen, Q. Sun, G.-Z. Liu et al., "Effect of miR-202-5pmediated ATG7 on autophagy and apoptosis of degenerative nucleus pulposus cells," European Review of Medical and Pharmacological Science, vol. 24, no. 2, pp. 517-525, 2020.

[19] X. B. Wang, H. Wang, H. Q. Long, D. Y. Li, and X. Zheng, "LINC00641 regulates autophagy and intervertebral disc degeneration by acting as a competitive endogenous RNA of miR-153-3p under nutrition deprivation stress," Journal of Cellular Physiology, vol. 234, no. 5, pp. 7115-7127, 2019.

[20] D. Zhan, M. Lin, J. Chen et al., "Hypoxia-inducible factor1alpha regulates PI3K/AKT signaling through microRNA-32-
$5 \mathrm{p} / \mathrm{PTEN}$ and affects nucleus pulposus cell proliferation and apoptosis," Experimental and Therapeutic Medicine, vol. 21, no. 6, p. 646, 2021.

[21] G. Li, X. Tang, H. Chen, W. Sun, and F. Yuan, "miR-148a inhibits pro-inflammatory cytokines released by intervertebral disc cells by regulating the p38/MAPK pathway," Experimental and Therapeutic Medicine, vol. 16, no. 3, pp. 2665-2669, 2018.

[22] C. W. Pfirrmann, A. Metzdorf, M. Zanetti, J. Hodler, and N. Boos, "Magnetic resonance classification of lumbar intervertebral disc degeneration," Spine (Phila Pa 1976), vol. 26, no. 17, pp. 1873-1878, 2001.

[23] Y. Li, Y. Gong, J. Ma, and X. Gong, "Overexpressed circRPL15 predicts poor survival and promotes the progression of gastric cancer via regulating miR-502-3p/OLFM4/STAT3 pathway," Biomedicine and Pharmacotherapy, vol. 127, Article ID 110219, 2020.

[24] H. Tsuchida, J. Hashimoto, E. Crawford, P. Manske, and J. Lou, "Engineered allogeneic mesenchymal stem cells repair femoral segmental defect in rats," Journal of Orthopaedic Research, vol. 21, no. 1, pp. 44-53, 2003.

[25] Y. Chen, L. Zhang, E. Li et al., "Long-chain non-coding RNA HOTAIR promotes the progression of osteoarthritis via sponging miR-20b/PTEN axis," Life Science, vol. 253, Article ID $117685,2020$.

[26] B. Han, "LncRNA LINC02418 regulates proliferation and apoptosis of non-small cell lung cancer cells by regulating miR-4677-3p/SEC61G," European Review Medical and Pharmacological Science, vol. 23, no. 23, pp. 10354-10362, 2019.

[27] X.-Q. Wang, W.-Z. Tu, J.-B. Guo et al., "A bioinformatic analysis of MicroRNAs' role in human intervertebral disc degeneration," Pain Medicine, vol. 20, no. 12, pp. 2459-2471, 2019.

[28] Y. Xi, J. Ma, and Y. Chen, "PTEN promotes intervertebral disc degeneration by regulating nucleus pulposus cell behaviors," Cell Biology International, vol. 44, no. 2, pp. 583-592, 2020.

[29] G. Xu, C. Liu, J. Jiang et al., "A novel mechanism of intervertebral disc degeneration: imbalance between autophagy and apoptosis," Epigenomics, vol. 12, no. 13, pp. 1095-1108, 2020.

[30] X. Y. Wang, L. Y. Jiao, J. L. He, Z. A. Fu, and R. J. Guo, "Parathyroid hormone 134 inhibits senescence in rat nucleus pulposus cells by activating autophagy via the mTOR pathway," Molecular Medicine Reports, vol. 18, no. 3, pp. 2681-2688, 2019.

[31] W. Zhang, Z. L. Li, and Q. Zhang, "Leptin inhibits apoptosis of nucleus pulposus cells via promoting autophagy," European Review of Medical and Pharmacological Sciences, vol. 22, no. 3, pp. 786-795, 2019.

[32] J. Sun, H. Chu, J. Ji, G. Huo, Q. Song, and X. Zhang, "Long non-coding RNA HOTAIR modulates HLA-G expression by absorbing miR-148a in human cervical cancer," International Journal of Oncology, vol. 49, no. 3, pp. 943-952, 2016.

[33] F. Xu and J. Zhang, "Long non-coding RNA HOTAIR functions as miRNA sponge to promote the epithelial to mesenchymal transition in esophageal cancer," Biomedicine and Pharmacotherapy, vol. 90, pp. 888-896, 2020.

[34] W. J. Wang, W. Yang, Z. H. Ouyang et al., "MiR-21 promotes ECM degradation through inhibiting autophagy via the PTEN/akt/mTOR signaling pathway in human degenerated NP cells," Biomedicine Pharmacotherapy, vol. 99, pp. 725-734, 2018. 
[35] L. Qingjuan, F. Xiaojuan, Z. Wei et al., "miR-148a-3p overexpression contributes to glomerular cell proliferation by targeting PTEN in lupus nephritis," American Journal of Physiology Cell Physiology, vol. 310, no. 6, pp. C470-C478, 2016.

[36] H. Zhang, Y. Wang, T. Xu et al., "Increased expression of microRNA-148a in osteosarcoma promotes cancer cell growth by targeting PTEN," Oncology Letter, vol. 12, no. 5, pp. 3208-3214, 2016.

[37] Y. Li, J. Nie, and P. Jiang, "Oleanolic acid mitigates interleukin-1beta-induced chondrocyte dysfunction by regulating miR-148-3p-modulated FGF2 expression," Journal of Gene Medicine, vol. 22, no. 5, Article ID e3169, 2021. 